\title{
Variations in Genome Size of Turnip Landraces from Two High-altitude Environments
}

\author{
Supriyo Basak, Guangyan Wang, Xudong Sun, and Yongping Yang ${ }^{1}$ \\ Key Laboratory for Plant Diversity and Biogeography of East Asia, Kunming Institute of Botany, \\ Chinese Academy of Sciences, 132\# Lanhei Road, Heilongtan, Kunming 650201, China; Germplasm \\ Bank of Wild Species, Kunming Institute of Botany, Chinese Academy of Sciences, 132\# Lanhei Road, \\ Heilongtan, Kunming, China 650201; and Institute of Tibetan Plateau Research at Kunming, \\ Kunming Institute of Botany, Chinese Academy of Sciences, 132\# Lanhei Road, Heilongtan, Kunming \\ 650201, China 650201
}

\begin{abstract}
ADDITIONAL INDEX wORDS. chromosome number, climatic variables, flow cytometry, intraspecies variation, Qinghai-Tibetan Plateau

ABstRact. Brassica rapa var. rapa (turnip) is considered a main source of food for the inhabitants of the QinghaiTibetan Plateau (QTP) and its adjacent highlands when other crops are scarce. The QTP ranges from lat. $25.59^{\circ} \mathrm{N}$ to $39.49^{\circ} \mathrm{N}$ and from long. $73.29^{\circ} \mathrm{E}$ to $104.40^{\circ} \mathrm{E}$, whereas the Yunnan Plateau ranges from lat. $20.00^{\circ} \mathrm{N}$ to $29.16^{\circ} \mathrm{N}$ and from long. $96.00^{\circ} \mathrm{E}$ to $110.19^{\circ} \mathrm{E}$. A comparison between the turnip landraces of two different plateau environments can provide a mechanistic insight into plant adaptation in highlands. The aim of this investigation was to understand the patterns in variation in genome size (GS) between the turnip landraces of two plateau environments. We used a wellestablished protocol to count chromosome number and performed propidium iodide flow cytometry to measure GS. No polyploidy was detected among the turnip landraces tested, and $15.5 \%$ variation in GS was observed between the landraces. No consistent pattern pertaining to GS variation emerged after the environmental variables were considered. Thus, we propose that such pattern may reflect the indirect effect of selection, random process, genetic drift, or some other factors on GS through interaction of life-form and phenotypic traits.
\end{abstract}

One of the goals of ecology is to explain the patterns of species abundance across different regions. Such patterns are assessed by investigating the phenotypic traits of congeneric taxa, such as seed size/number, plant height, and specific leaf area. Genome size (GS) is the amount of DNA in an unreplicated, basic, and gametic chromosome set; the regional abundance of a species can be predicted by using GS as a proxy for plant phenotypic traits because GS covaries with the rate of cell division and cell size (Knight and Beaulieu, 2008). Hence, GS is proposed as a key determinant constraining plant fitness over ecological time (Knight et al., 2005). For example, researchers who studied large-scale cytotype distribution (McAllister et al., 2015) hypothesized that higher GS plants adapt better in high altitudes than lower GS plants. However, the results of some studies are inconsistent with this hypothesis (Guo et al., 2016). For instance, Bennett et al. (1982) indicated that lower GS plants have increased tolerance in highlands. These contrasting ideas illustrate that higher GS may be a necessary but not critical to life in high-elevation areas (Knight and Ackerly, 2002). However, this hypothesis remains to be validated experimentally for flowering plants adapted to cultivation in the Qinghai-Tibetan Plateau (QTP) and the Yunnan Plateau (YP), which may strengthen knowledge in plant domestication in the highlands.

QTP is one of the world's largest biodiversity hotspots. It has an average altitude of more than $4500 \mathrm{~m}$ and is thus called "the roof of the world." QTP had undergone a dramatic change in

Received for publication 26 Jan. 2018. Accepted for publication 8 Mar. 2018. We thank Yuanwen Duan for statistical advice. This work was financially supported by Yunnan Postdoctoral Grants to S. Basak, Major State Basic Research Development Program (2010CB951704), and National Natural Science Foundation of China (NSFC) (41271058) to Y. P. Yang.

${ }^{1}$ Corresponding author. E-mail: yangyp@mail.kib.ac.cn. climate during the past million years, such as conversion of forests to grasslands; formation of glaciers and desserts; arid and chilly climate (Wu et al., 2001). Meanwhile, YP, situated at an average elevation of $2000 \mathrm{~m}$, is known as a hotspot for plant diversity (Ruth et al., 2008). In QTP, environmental extremes have led to the evolution of many flowering plants at the intraspatial scale, whereas, flora in YP exhibits particularly strong spatial and ecological isolation because of its unique landscape and climate. Intraspatial comparative phylogenomic study between the two areas shows two distinct populations resulting from the fragmentation of two plateaus by periods of glaciation and subsequent interruption in gene flow (Li et al., 2011). Moreover, species adapted to QTP show higher genetic diversity than in YP because of their enhanced tolerance to extreme climate ( $\mathrm{Li}$ et al., 2011). Low temperature, high ultraviolet radiation, and drought severely affect the physiology of highland vegetation and shape GS by imposing selection pressure on the physiology and life history traits correlated to GS (Knight and Ackerly, 2002). Unfortunately, intraspatial GS variation among landraces that adapted to cultivation in QTP and YP is rarely investigated. In this study, we investigated GS variation between the landraces grown in QTP and YP at the intraspatial scale.

In flowering plants, intraspatial GS research has considerable gaps. Although there are several studies reporting the existence of intraspatial genome plasticity, these results were either doubted or rejected on taxonomical and technological grounds. Feulgen densitometry was used in the late 19th century to compare GS variation; however, this method was not appropriate to assess intraspecies GS variation. This has changed because flow cytometry became available, as it provides the necessary accuracy to obtain estimates of intraspecies GS variation. But usage of internal standards, consideration of cytosolic inhibitors, and documentation of double peak by cochopping plant specimen are absolutely 
important for flow cytometry to provide evidence for intraspecies GS variation. Hence, there was necessity to generate standard operating procedures for identifying intraspecies variation of GS throughout the world. During the last decade, several groups developed standardized protocols for measuring intraspecies GS variation (Doležel and Bartoš, 2005). Recent investigation based on flow cytometry confirmed that true intraspatial variation exists among angiosperms, but the level of intraspatial variation that can be accommodated by a species remains unclear (Benor et al., 2011). Moreover, whether GS variation is governed by a selection process is unknown so far. Although sequencing is the most powerful technique for the investigation of adaptive evolution in angiosperms, GS calculated from sequencing data is still prone to errors because of the misassembly of repetitive genomes. Despite the availability of GS estimates for 220 species of 4060 members from Brassicaceae family (5\%) (Bennett and Leitch, 2011), intraspatial GS information remains scarce (but see Long et al.,
2013). The mechanism by which GS has been shaped by ecological and evolutionary forces among cultivated members of this family is unknown.

Turnip is an important biennial, outcrossing, mesopolyploid herbaceous plant in the Brassicaceae family and is native throughout Europe, Russia, Central Asia, and Near East. Europe has been proposed to be the center of origin. In the alpine regions of QTP, turnip was widely used as a food source when other crops are scarce. The highlands of QTP which range in altitude between 3000 and $4500 \mathrm{~m}$ are characterized by strong ultraviolet-B radiation and low accessibility of water (Liu et al., 2012). Turnip is able to germinate during cold and drought. It also grows at high elevation (up to $1000 \mathrm{~m}$ in Kumaon Himalaya, India) in tropical countries, such as India, Nepal, Bhutan, Bangladesh, Sri Lanka, and Australia.

Because of its AA genome, turnip is one of the ancestor species of Brassica juncea $(2 n=36$, genome $\mathrm{AABB})$ and

Table 1. Information of the collection sites and distinguishing morphological characteristics of 23 Brassica rapa var. rapa landraces. From this table, it is evident that Yunnan Plateau (YP) landraces selected in this investigation are relatively from lower latitude depicting the geographical distribution of YP compared with Qinghai-Tibetan Plateau (QTP). Moreover, morphological description of leaf length, aboveground biomass, tuber diameter, and underground biomass for 23 landraces from the two plateau environments demonstrate that YP landraces are bigger than QTP landraces.

\begin{tabular}{|c|c|c|c|c|c|c|c|c|c|c|}
\hline $\begin{array}{l}\text { Serial } \\
\text { no. }\end{array}$ & Environment & $\begin{array}{c}\text { Accession } \\
\text { no. }^{\mathrm{z}}\end{array}$ & Origin $^{y}$ & $\begin{array}{c}\text { Latitude } \\
\left({ }^{\circ} \mathrm{N}\right)\end{array}$ & $\begin{array}{c}\text { Longitude } \\
\left({ }^{\circ} \mathrm{E}\right)\end{array}$ & $\begin{array}{c}\text { Altitude } \\
(\mathrm{m})\end{array}$ & $\begin{array}{l}\text { Leaf length } \\
{[\text { mean } \pm \text { SE }} \\
(\mathrm{cm})]^{\mathrm{x}}\end{array}$ & $\begin{array}{l}\text { Aboveground } \\
\text { biomass } \\
{[\text { mean } \pm \mathrm{SE}} \\
(\mathrm{kg})]^{\mathrm{x}} \\
\end{array}$ & $\begin{array}{c}\text { Tuber diam } \\
{[\text { mean } \pm \text { SE }} \\
(\mathrm{mm})]^{\mathrm{x}}\end{array}$ & $\begin{array}{c}\text { Under-ground } \\
\text { biomass } \\
{[\text { mean } \pm \text { SE }} \\
(\mathrm{kg})]^{\mathrm{x}} \\
\end{array}$ \\
\hline 2 & & KTRG-B-04 & $\mathrm{F}$ & 31.32 & 95.21 & 3,643 & $75.60 \pm 6.02$ & $0.27 \pm 0.05$ & $92.97 \pm 1.17$ & $0.37 \pm 0.11$ \\
\hline 3 & & KTRG-B-05 & $\mathrm{H}$ & 35.40 & 97.35 & 4,223 & $65.42 \pm 2.70$ & $0.19 \pm 0.04$ & $69.38 \pm 9.27$ & $0.15 \pm 0.04$ \\
\hline 4 & & KTRG-B-06 & B & 33.22 & 97.6 & 3,825 & $77.54 \pm 5.61$ & $0.28 \pm 0.03$ & $87.56 \pm 5.44$ & $0.24 \pm 0.43$ \\
\hline 6 & & KTRG-B-14 & $\mathrm{J}$ & 29.39 & 100.04 & 3,416 & - & - & - & - \\
\hline 7 & & KTRG-B-17 & $\mathrm{D}$ & 29.36 & 91.06 & 3,658 & $45.74 \pm 2.17$ & $0.05 \pm 0.01$ & $92.07 \pm 7.93$ & $0.26 \pm 0.04$ \\
\hline 8 & & KTRG-B-19 & $\mathrm{O}$ & 27.89 & 102.26 & 1,542 & $60.23 \pm 3.48$ & $0.16 \pm 0.05$ & $73.19 \pm 1.18$ & $0.18 \pm 0.08$ \\
\hline 9 & & KTRG-B-49 & $\mathrm{L}$ & 30.55 & 96.62 & 4,155 & $81.30 \pm 5.80$ & $0.29 \pm 0.09$ & $89.02 \pm 8.82$ & $0.35 \pm 0.14$ \\
\hline 10 & & KTRG-B-57 & I & 29.35 & 90.74 & 3,594 & $50.66 \pm 4.75$ & $0.08 \pm 0.02$ & $97.15 \pm 8.36$ & $0.38 \pm 0.10$ \\
\hline Summ & ary statistics & & & 31.04 & 90.74 & 3,420 & $63.24 \pm 2.54$ & $0.21 \pm 0.02$ & $86.54 \pm 3.43$ & $0.29 \pm 0.03$ \\
\hline 5 & & KTRG-B-13 & M & 28.02 & 99.34 & 2,320 & $79.16 \pm 9.71$ & $0.45 \pm 0.21$ & $114.24 \pm 1.36$ & $0.65 \pm 0.27$ \\
\hline 6 & & KTRG-B-15 & Q & 26.59 & 100.12 & 2,730 & $68.08 \pm 8.77$ & $0.42 \pm 0.20$ & $126.25 \pm 3.80$ & $1.23 \pm 0.71$ \\
\hline 7 & & KTRG-B-16 & $\mathrm{R}$ & 26.45 & 100.16 & 2,450 & - & - & - & - \\
\hline 8 & & KTRG-B-25 & $\mathrm{P}$ & 26.45 & 99.91 & 2,227 & $80.16 \pm 5.66$ & $0.55 \pm 0.12$ & $93.51 \pm 1.89$ & $0.59 \pm 0.30$ \\
\hline 9 & & KTRG-B-55 & $\mathrm{N}$ & 26.95 & 100.20 & 2,622 & $73.00 \pm 5.34$ & $0.54 \pm 0.14$ & $127.53 \pm 2.53$ & $0.99 \pm 0.44$ \\
\hline 10 & & KTRG-B-65A & G & 27.28 & 100.85 & 2,248 & $92.90 \pm 5.74$ & $0.48 \pm 0.07$ & $139.83 \pm 1.53$ & $0.62 \pm 0.12$ \\
\hline 11 & & KTRG-B-61 & G & 27.28 & 100.85 & 2,248 & $100.86 \pm 5.17$ & $0.53 \pm 0.10$ & $120.26 \pm 2.32$ & $0.87 \pm 0.31$ \\
\hline 12 & & KTRG-B-65B & G & 27.28 & 100.85 & 2,248 & - & - & - & - \\
\hline 13 & & KTRG-B-54 & G & 27.28 & 100.85 & 2,248 & $73.08 \pm 7.02$ & $0.56 \pm 0.17$ & $103.87 \pm 1.98$ & $0.51 \pm 0.22$ \\
\hline \multicolumn{2}{|c|}{ Summary statistics } & & & 27.04 & 100.43 & 2,387 & $76.96 \pm 2.81$ & $0.46 \pm 0.04$ & $105.22 \pm 7.42$ & $0.64 \pm 0.09$ \\
\hline
\end{tabular}

$\mathrm{SE}=$ standard error.

${ }^{\mathrm{z}}$ KTRG stands for accession number of B. rapa var. rapa germplasm (Kunming Turnip Research Group).

${ }^{\mathrm{y}} \mathrm{A}=\mathrm{Aba}$ Tibetan and Qiang Autonomous Prefecture, Sichuan Province, B = Chengduo County, Yushu Prefecture, Qinghai Province, C = Huize County, Qujing city, Yunnan Province, D = Lhasa City, Tibetan Autonomous Region, E = Lianhe Village, Small Zhong Dian Town, Shangri-la City, Yunnan Province, F = Nangqian County, Yushu Prefecture, Qinghai Province, G = Ninlang, Lijiang City, Yunnan Province, H = Qumalai County, Yushu Prefecture, Qinghai Province, I = Qushui County, Lhasa City, Tibet, J = Reda Township, Xiangcheng County, Sichuan Province, $\mathrm{K}=$ Shangri-la City, Yunnan Province, $\mathrm{L}=$ Tongqi Town, Changdu City, Tibet, $\mathrm{M}=$ Weixi County, Diqing Tibetan Autonomous Prefecture, Yunnan Province, $\mathrm{N}=$ Wenhai County, Lijiang city, Yunnan Province, $\mathrm{O}=$ Xichang City, Sichuan Province, $\mathrm{P}=\mathrm{Xunnan}$ Town, Dali County, Yunnan Province, Q = Yuhu Village, Lijiang city, Yunnan Province, R = Zhongyi Village, Qihe County, Lijiang City, Yunnan Province.

${ }^{\mathrm{x}}$ Five individuals of each landrace were analyzed for phenotypic studies. 
Brassica napus $(2 n=38$, genome AACC). Unveiling the intraspatial GS variation in turnip may facilitate research on genome evolution and applied research on crop improvement across cultivated Brassica species. We speculate that turnip landraces from QTP (Qinghai, Sichuan, and Tibet) possess smaller GS than the turnip landraces of YP (Yunnan Province). Thus, we investigated the GS of turnip landraces from two plateaus to evaluate their differential adaptation.

\section{Materials and Methods}

Plant material. Seeds of all turnip landraces used in this investigation were collected from 2015 to 2017. Ten landraces were collected from QTP including four landraces from Qinghai Province, three landraces from Sichuan Province, and three landraces belonging to Tibet autonomous region. Thirteen landraces were collected from Yunnan Province (Table 1). Seeds were grown in the germplasm nursery of Kunming Institute of Botany, Yunnan, China (lat. $25.02^{\circ} \mathrm{N}$, long. $102.68^{\circ} \mathrm{E}$, and altitude $1900 \mathrm{~m}$ ). The taxonomic identification was carried out by Yongping Yang. Landraces showed marked morphological differences, with YP being generally larger and also producing bigger turnips than QTP landrace (Table 1; Supplemental Fig. 1). Germinated root tips were used for determination of somatic chromosome number. Glycine max 'Polanka' [soybean (Doležel et al., 1994)] was used as the internal reference standard $(2 \mathrm{C}=2.50 \mathrm{pg})$, which was collected on request and raised in a greenhouse. Turnip landraces were raised from seeds in 16-cm-wide and 14.5-cmdeep round, plastic containers in soils on greenhouse benches in a temperature range of $22.2 \pm 6^{\circ} \mathrm{C}$ during the day and $18 \pm 3{ }^{\circ} \mathrm{C}$ at night. Young leaves from the grown landraces and standards were used for the estimation of GS.

SAMPLE PREPARATION AND FLOW CYTOMETRY STUDY. Investigation of GS was performed in a flow cytometer as described previously (Guo et al., 2016). In brief, young leaves were collected from each turnip seedling and kept on ice until processing, which occurred within one h of sample collection. For each turnip individual, $0.5-\mathrm{cm}^{2}$ sections were taken and kept in a plastic petri dish containing $800 \mu \mathrm{L}$ of prechilled woody plant buffer [WPB (Loureiro et al., 2007)] [0.2 $\mathrm{mol} \cdot \mathrm{L}^{-1}$ Tris-HCl, $4 \mathrm{mmol} \cdot \mathrm{L}^{-1} \mathrm{MgCl}_{2}, 6 \mathrm{H}_{2} \mathrm{O}, 2 \mathrm{mmol} \cdot \mathrm{L}^{-1} \mathrm{EDTANa}_{2} \cdot 2 \mathrm{H}_{2} \mathrm{O}$, $86 \mathrm{mmol} \cdot \mathrm{L}^{-1} \mathrm{NaCl}, 10 \mathrm{mmol} \cdot \mathrm{L}^{-1}$ sodium metabisulfite, $1 \% \mathrm{PVP}-10$ (Thermo Fisher Scientific, Shanghai, China), 1\% (v/v) Triton X-100 (Thermo Fisher Scientific), and $\mathrm{pH} 7.5]$. A $0.25-\mathrm{cm}^{2}$ section of soybean leaf was added to the petri dish for internal standardization. Leaf tissues were chopped using a sterile stainless steel razor blade. An additional $800 \mu \mathrm{L}$ of WPB was added after chopping. The sample was filtered through a $30-\mu \mathrm{m}$ nylon mesh (CellTrics ${ }^{\circledR}$; Sysmex Partec, Görlitz, Germany). The supernatant was stained with a solution containing $2 \mathrm{mg} \cdot \mathrm{mL}^{-1}$ propidium iodide [PI (Sigma, St. Louis, MO)] and $50 \mu \mathrm{g} \cdot \mathrm{mL}^{-1}$ RNase A (Sigma). The suspension was acquired in a flow cytometer (Sysmex Partec) equipped with a solid-state laser for green light emission at $532 \mathrm{~nm}$. Histograms were analyzed by FlowJo software (version 7.6.1; Tree Star, Ashland, OR). Six individuals from each landrace were analyzed for estimation of GS and three different leaves of each individual were used for flow cytometric estimation. We followed strict criteria to obtain precise and stable difference in GS at the intraspatial scale, such as the consistent use of an internationally accepted internal standard for all measurements, repeating the measurements on three different days, checking for the presence of metabolic compounds that affect PI staining, and verification of any GS variation by looking for double peak of two cochopped samples differing in GS.

Test FOR INHIBITORs. Plant secondary metabolites may interfere with PI staining and may result in slight differences between the estimates. Thus, we tested the inhibitors to ascertain the effect of the cytosolic compounds of turnip on the nuclear fluorescence of soybean nuclei. Treatment A consisted of PI-stained nuclei from the independently processed and stained leaves of turnip and soybean. Turnip and soybean were coprocessed and stained with PI, designated as treatment B. Data were collected for three replicate experiments. The comparative study of nuclear fluorescence of soybean nuclei from independently processed soybean leaves and simultaneously processed turnip-soybean leaves gave evidence of no inhibitors.

Contribution OF ECOGEOGRAPHical VARIABLES to GS. To assess the contribution of environmental variables affecting GS, geographical and bioclimatic variables were considered.

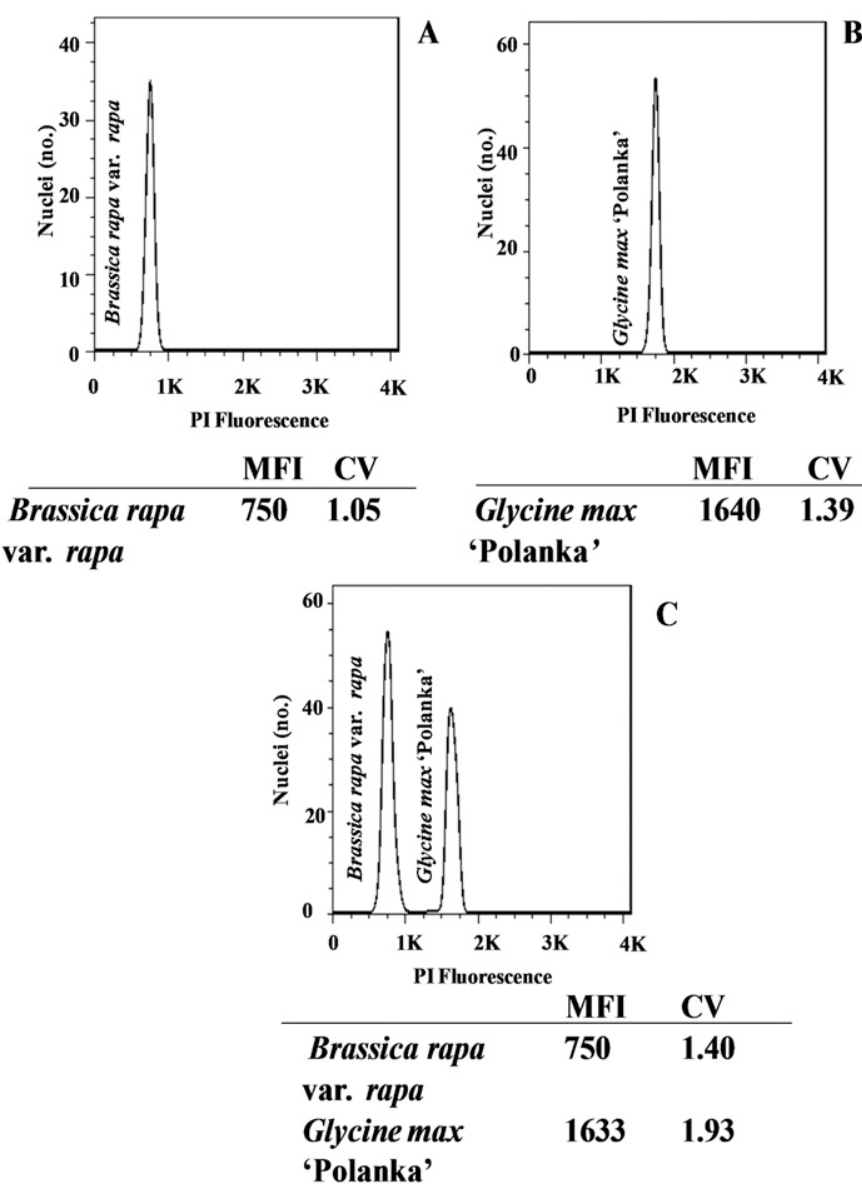

Fig. 1. Histogram of mean fluorescence intensity (MFI) of Brassica rapa var. rapa and Glycine max 'Polanka' nuclei isolated with woody plant buffer. (A and B) Leaves were treated individually or (C) simultaneously processed [cochopped ] and stained with propidium iodide (PI). Analyses confirmed that genome size is unaffected by secondary metabolites of the leaves; $\mathrm{x}$-axis represents relative fluorescence and y-axis represents number of nuclei. Mean fluorescence intensity of 1000 was represented by $1 \mathrm{k}$ in $\mathrm{x}$-axis. $\mathrm{CV}=$ coefficient of variation. 
Information on latitude, longitude, and altitude was noted during the time of collection of the landraces. Bioclimatic variables are a summary of mean temperature and precipitation, which describe the environment of 23 landrace collection sites. Within R [version 3.02 (R Core Team, 2013)], we downloaded raster-formatted mean values of 19 climatic variables for 23 places with $\approx 1-\mathrm{km}^{2}$ spatial resolutions from the WorldClim dataset (Hijmans et al., 2005). Principal component analysis (PCA) was performed based on the geographical, bioclimatic variables, and GS to know about niche differentiation among landraces from the two plateau environments.

Chromosome observation. Chromosome numbers were counted in the actively growing root tips of 12 turnip landraces. Mature seeds were allowed to sprout for 2-3 d in the moistened petri plate maintained at $22{ }^{\circ} \mathrm{C}$ incubator. Roots (1-2-cm long) were collected in the morning at different time intervals and were pretreated with a saturated solution of p-dichlorobenzene $(2 \mathrm{~h}$, room temperature), fixed in a freshly prepared 3:1 mixture of absolute ethanol and acetic acid at $4{ }^{\circ} \mathrm{C}$, and squashed in a drop of lacto-propionic orcein under cover slips, which were sealed with nail polish to make a temporary slide. The cover slips were separated from the slide by dipping in liquid nitrogen. The slides were then dehydrated in absolute ethanol and mounted in DPX (distyrene, plasticizer, and xylene). Well-spread mitoses were photographed from these slides and chromosomes were counted. For each landrace, 10 and 15 counts were made involving counting squashes prepared from three to six roots.

Statistical analysis. One way analysis of variance (ANOVA) at a significance level of $1 \%(\alpha=0.01)$ was used to evaluate difference in GS among landraces, and Tukey's honest significant difference test was used to evaluate differences among groups of landraces in SPSS (version 15.0; IBM Corp., Armonk, NY). The relationship between GS and bioclimatic variables was assessed by applying Pearson's correlation coefficient and PCA.

\section{Results and Discussion}

Flow CYTOMetry. We compared the nuclear fluorescences of independently processed soybean nuclei and coprocessed turnip and soybean nuclei of all landraces to illustrate that the secondary metabolites of turnip have no inhibitory effects on PI staining. The average coefficients of variation (CV) were $1.05 \%$ (turnip) and $1.39 \%$ (soybean) for independently processed samples, whereas the average CV were $1.40 \%$ (turnip) and $1.93 \%$ (soybean) for coprocessed samples. These values were within the acceptable limit $(\mathrm{CV}<2 \%)$, and thus, the GS estimates were reliable (Fig. 1).

One way ANOVA results showed that the GSs $(2 \mathrm{C})$ of the 23 landraces

$\mathrm{SE}=$ standard error. variance at $P \leq 0.01$. varied significantly $\left(\mathrm{F}_{22,487}=10.06, P \leq 0.01\right)$ and categorized the landraces into five statistical groups (Table 2). Statistically significant variation in GS was observed for landraces belonging to QTP $\left(\mathrm{F}_{9,196}=7.50, P \leq 0.01\right)$ and $\mathrm{YP}\left(\mathrm{F}_{12,290}=2.56\right.$, $P \leq 0.01)$. The lowest GS (2C) was observed in landraces [KTRG-B-03 $(2 \mathrm{C}=1.03 \pm 0.02 \mathrm{pg}), \mathrm{KTRG}-\mathrm{B}-06(2 \mathrm{C}=1.03 \pm$ $0.01 \mathrm{pg})$, and KTRG-B-08 $(2 \mathrm{C}=1.03 \pm 0.01 \mathrm{pg})]$ from QTP. The highest GS (2C) was found for KTRG-B-02A [2C=1.19 \pm $0.01 \mathrm{pg}($ mean $\pm \mathrm{SE})]$ adapted to cultivation in YP (Table 2). The range of variation of GS was found to be $2 \mathrm{C}=1.03-1.11 \mathrm{pg}$, $7.76 \%$ for QTP and $2 \mathrm{C}=1.11-1.19 \mathrm{pg}, 7.20 \%$ for YP. The mean GS (2C) for turnip was found to be $1.11 \pm 0.01 \mathrm{pg}$ with 1.15 -fold $(\approx 15.5 \%$ ) variation.

Simultaneous processing of landraces belonging to lower GS (KTRG-B-03, KTRG-B-06, and KTRG-B-08) of QTP and the highest GS race (KTRG-B-02A) of YP produced double peaks in the flow cytometer (Fig. 2), confirming true difference in GS among the landraces (Table 2). The appearance of separate peaks in simultaneous analysis is the most straightforward evidence of the differences among GSs (Greilhuber et al., 2007). Several authors have performed experiments to show the true difference of GS by double peaks (Achigan-Dako et al., 2008; Benor et al., 2011; Tatum et al., 2006).

Climate change may play an important role in the biogeographical distribution of angiosperms. Plants of alpine habitat adapted a series of mechanisms to counter environmental

Table 2. Mean values for nuclear DNA content (2C) and coefficient of variation (CV) of 23 Brassica rapa var. rapa landraces obtained by flow cytometry analysis using Glycine max 'Polanka' (2C = $2.50 \mathrm{pg}$ ) as internal reference standard. Five genome size (GS) groups were found among landraces with the mean $2 \mathrm{C}$ value of $1.11 \pm 0.01 \mathrm{pg}$.

\begin{tabular}{lclcc}
\hline Environment & Serial no. & Accession no. & 2C GS [mean $\pm \mathrm{sE}(\mathrm{pg})]^{\mathrm{z}}$ & CV (\%) \\
\hline Qinghai-Tibetan Plateau & 1 & KTRG-B-03 & $1.03 \pm 0.02 \mathrm{a}$ & 1.43 \\
& 2 & KTRG-B-04 & $1.04 \pm 0.01 \mathrm{ab}$ & 1.38 \\
& 3 & KTRG-B-05 & $1.03 \pm 0.01 \mathrm{a}$ & 1.75 \\
& 4 & KTRG-B-06 & $1.03 \pm 0.01 \mathrm{a}$ & 1.98 \\
& 5 & KTRG-B-08 & $1.03 \pm 0.02 \mathrm{a}$ & 1.24 \\
& 6 & KTRG-B-14 & $1.11 \pm 0.01 \mathrm{abcde}$ & 1.65 \\
& 7 & KTRG-B-17 & $1.09 \pm 0.01 \mathrm{abcde}$ & 1.93 \\
& 8 & KTRG-B-19 & $1.08 \pm 0.01 \mathrm{abcd}$ & 1.64 \\
9 & KTRG-B-49 & $1.06 \pm 0.01 \mathrm{abc}$ & 1.40 \\
Yunnan Plateau & 10 & KTRG-B-57 & $1.11 \pm 0.01 \mathrm{abcde}$ & 1.23 \\
& 11 & KTRG-B-01 & $1.18 \pm 0.02 \mathrm{e}$ & 1.56 \\
& 12 & KTRG-B-02A & $1.19 \pm 0.01 \mathrm{e}$ & 1.78 \\
& 13 & KTRG-B-02B & $1.13 \pm 0.02 \mathrm{bcde}$ & 1.99 \\
& 14 & KTRG-B-09 & $1.14 \pm 0.03 \mathrm{cde}$ & 1.45 \\
& 15 & KTRG-B-13 & $1.17 \pm 0.03 \mathrm{de}$ & 1.53 \\
16 & KTRG-B-15 & $1.17 \pm 0.03 \mathrm{de}$ & 1.94 \\
& 17 & KTRG-B-16 & $1.13 \pm 0.02 \mathrm{cde}$ & 1.60 \\
18 & KTRG-B-25 & $1.13 \pm 0.01 \mathrm{bcde}$ & 1.34 \\
& 19 & KTRG-B-55 & $1.12 \pm 0.01 \mathrm{abcde}$ & 1.43 \\
20 & KTRG-B-65A & $1.13 \pm 0.01 \mathrm{bcde}$ & 1.85 \\
& 21 & KTRG-B-61 & $1.13 \pm 0.01 \mathrm{bcde}$ & 1.53 \\
& 22 & KTRG-B-65B & $1.11 \pm 0.01 \mathrm{abcde}$ & 1.32 \\
& KTRG-B-54 & $1.17 \pm 0.01 \mathrm{de}$ & 1.65
\end{tabular}

${ }^{\mathrm{z}}$ Holoploid GSs were determined with propidium iodide as the fluorochrome. Means within a column followed by the same letter are not significantly different via one way analysis of 

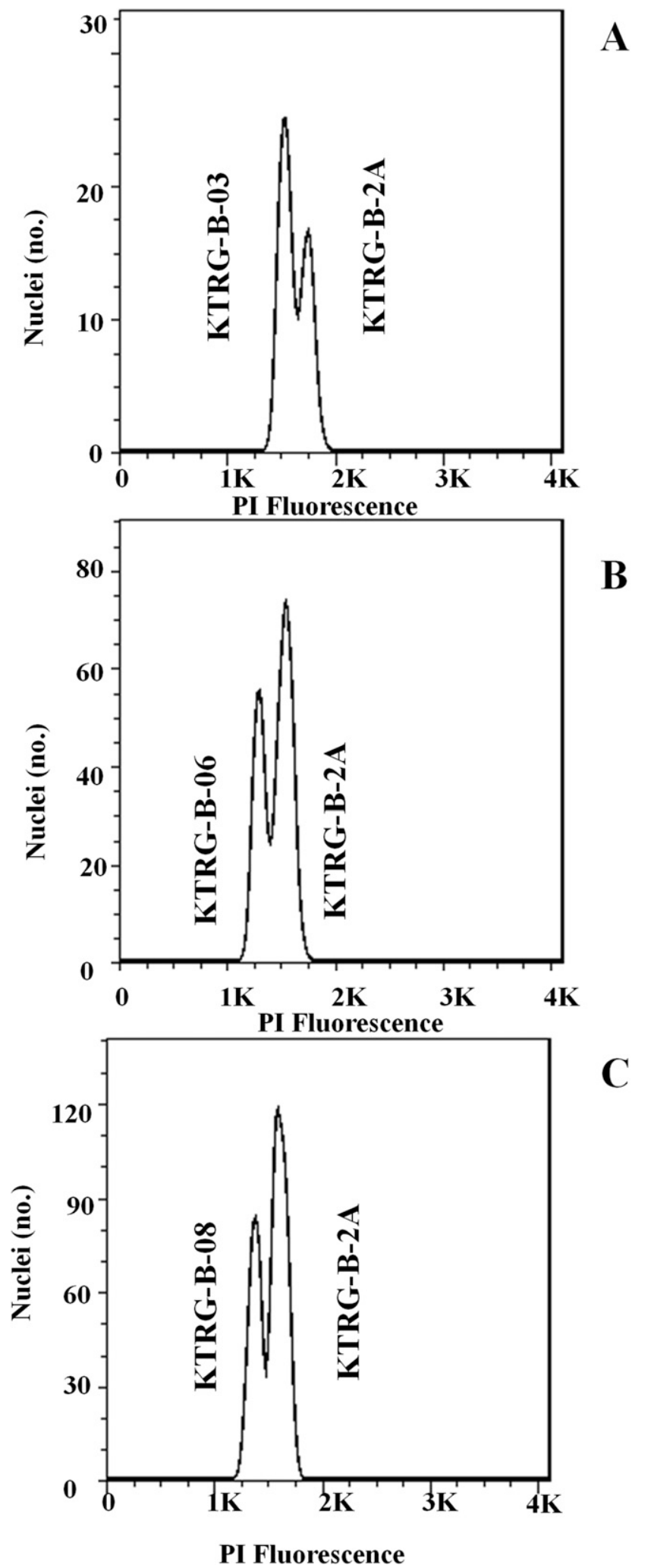

Fig. 2. Histogram of double peaks or bimodal peaks representing true intraspatial genome size (GS) variation between Qinghai-Tibetan Plateau (QTP) and Yunnan Plateau Brassica rapa var. rapa landraces. (A) KTRG-B-03 (small genome) with KTRG-B-2A (big genome), (B) KTRG-B-06 (small genome) with KTRG-B-2A (big genome), and (C) KTRG-B-08 (small genome) with KTRG-B-2A (big genome). These three results demonstrate that GS is lesser for B. rapa var. rapa landraces from QTP (KTRG-B-03, KTRG-B-06, and KTRG-B-08). Mean fluorescence intensity of 1000 was represented by $1 \mathrm{k}$ in $\mathrm{X}$-axis. $\mathrm{PI}=$ propidium iodide. stress created by low temperature and precipitation. Turnip landraces adapted to QTP experience comparatively lesser temperature (from 7 to $15^{\circ} \mathrm{C}$ ) and precipitation (from 100 $300 \mathrm{~mm}$ to $500-800 \mathrm{~mm}$ ) compared with YP because of its special geographical positioning (Piao et al., 2003). Thus, we hypothesized that natural selection may play a significant role for lower GS of turnip landraces from QTP. Geographical and climatic variables were correlated significantly with GS based on Pearson's correlations (Table 3) considering both plateau environments. For example, significant correlation of GS with geographical and bioclimatic variables was significant for the entire landraces including latitude, temperature $(\mathrm{BIO} 1, \mathrm{BIO} 2, \mathrm{BIO} 3, \mathrm{BIO} 4, \mathrm{BIO} 6, \mathrm{BIO} 7, \mathrm{BIO} 9$, and $\mathrm{BIO} 11$ ), and precipitation (BIO12, BIO13, BIO16, and BIO18). When we considered correlation analysis of each of the individual plateau environments separately, the pattern was inconsistent. For example, GS was correlated significantly with latitude for QTP but not for YP (Table 3). This kind of inconsistency in correlation analyses between GS and biogeographic variables from two plateau environments tells that GS variation may not be controlled by natural selection. Previous investigator failed to find convincing evidence to demonstrate strong effect of natural selection on GS (Díez et al., 2013).

To illustrate the effect of other factors governing GS variation, we performed PCA to cluster landraces based on complete dataset; i.e., GS information plus geographical and bioclimatic variables (Fig. 3). The first principal component explained $59.62 \%$ of the total variance and the second component explained $22.77 \%$ of the variance in PCA, confirming acceptable grouping among landraces. In PCA, the direction of the arrow of bioclimatic variables exhibited landrace usage (Díez et al., 2013). Most of the bioclimatic variables were directed toward landraces belonging to YP, depicting differences in landrace usage between the two plateau environments. Moreover, landraces were found to be scattered irrespective of GS in the climatic space of PCA, demonstrating ecological flexibility. Thus, GS variation among turnip landraces may be governed by artificial selection, random process, genetic drift, or some other factors through interaction of life history and phenotypic traits.

Chromosome SPRead. Chromosomal number variation may have contributed to the proposed GS variability among landraces from the two plateau environments. Chromosomal numbers were determined for six landraces, which showed GS difference from each plateau environment to minimize this possibility. Sizes of chromosomes were found to be very small for both environments (Fig. 4). Metaphase counts of QTP and YP showed that these landraces were euploids with the chromosome number $2 n=2 x=20$. Thus, it can be concluded that GS variation was not the result of chromosomal abnormality.

Deletions/insertional mutagenesis (Bennetzen et al., 2005) and illegitimate recombination among QTP and YP turnips may lead to GS variation. Deletion/insertional mutagenesis might occur because of differences in the content of repetitive DNA, caused by the proliferation of transposable element as reported earlier in wild barley [Hordeum spontaneum (Kalendar et al., 2000)] and maize [Zea mays (Messing et al., 2004)]. Double-stranded break (DSB) may occur in the genome of QTP turnip as it is exposed to high ultraviolet radiation at high altitudes. During the repair of these DSB (illegitimate recombination) via nonhomologous end 
Table 3. Relationship of Brassica rapa var. rapa genome size with environmental factors as revealed by correlation analyses.

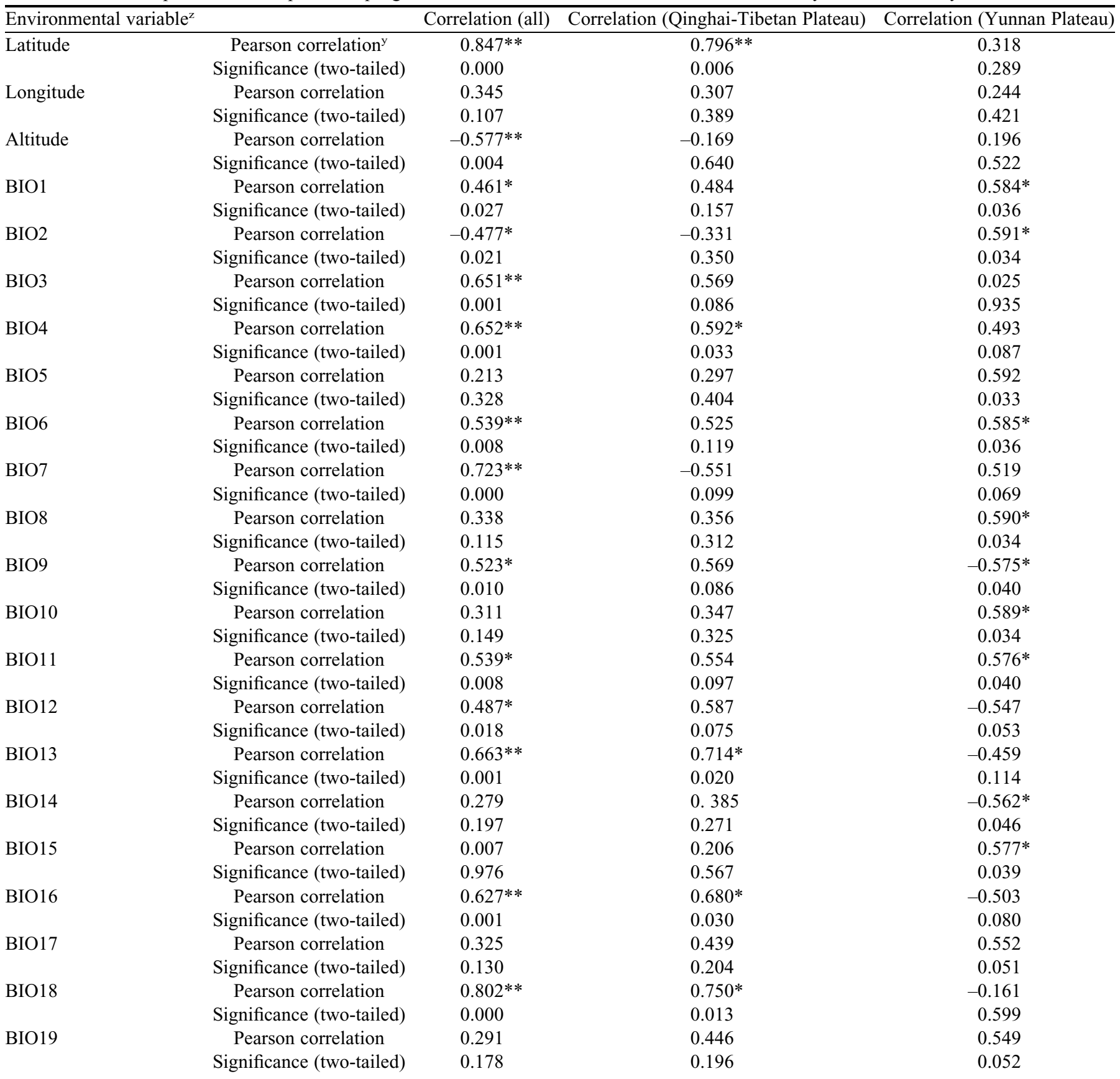

${ }^{\mathrm{z}} \mathrm{BIO} 1$ = mean annual temperature; $\mathrm{BIO} 2$ = mean diurnal range [mean of monthly (maximum temperature - minimum temperature) $]$; $\mathrm{BIO} 3=$ isothermality $[(\mathrm{BIO} 2 / \mathrm{BIO} 7) \times 100] ; \mathrm{BIO} 4=$ temperature seasonality $(\mathrm{SD} \times 100)$; $\mathrm{BIO} 5=$ maximum temperature of warmest mo.; $\mathrm{BIO} 6=$ minimum temperature of coldest mo.; $\mathrm{BIO} 7=$ range annual temperature $(\mathrm{BIO} 5-\mathrm{BIO} 6)$; $\mathrm{BIO} 8=$ mean temperature of wettest quarter; $\mathrm{BIO} 9=$ mean temperature of driest quarter; $\mathrm{BIO} 10=$ mean temperature of warmest quarter; BIO11 = mean temperature of coldest quarter; $\mathrm{BIO} 12$ = annual precipitation; $\mathrm{BIO} 13$ = precipitation of wettest mo.; BIO14 = precipitation of driest mo.; BIO15 = precipitation seasonality $(\mathrm{cv})$; $\mathrm{BIO} 16$ = precipitation of wettest quarter; $\mathrm{BIO} 17$ = precipitation of driest quarter; $\mathrm{BIO} 18=$ precipitation of warmest quarter; $\mathrm{BIO} 19=$ precipitation of coldest quarter.

${ }^{\mathrm{y}}$ Pearson correlation coefficient based on the total 23 landraces. Significance levels are denoted by asterisks: ${ }^{*} P \leq 0.05, * * P \leq 0.01$.

joining, small-scale deletion occurs. This process was observed experimentally in Arabidopsis thaliana (Kirik et al., 2000). Unequal homologous recombination is another mechanism of GS variability where increase in size and rate of deletion of DNA fragments (in bps) relative to insertion was proposed (Bennetzen et al., 2005).

\section{Conclusion}

We observed 1.15-fold variations between the GSs obtained from the two highland environments. GS variation was not correlated with environmental parameters. Thus, we conclude that differences among GSs reflect the indirect effect of selection. 


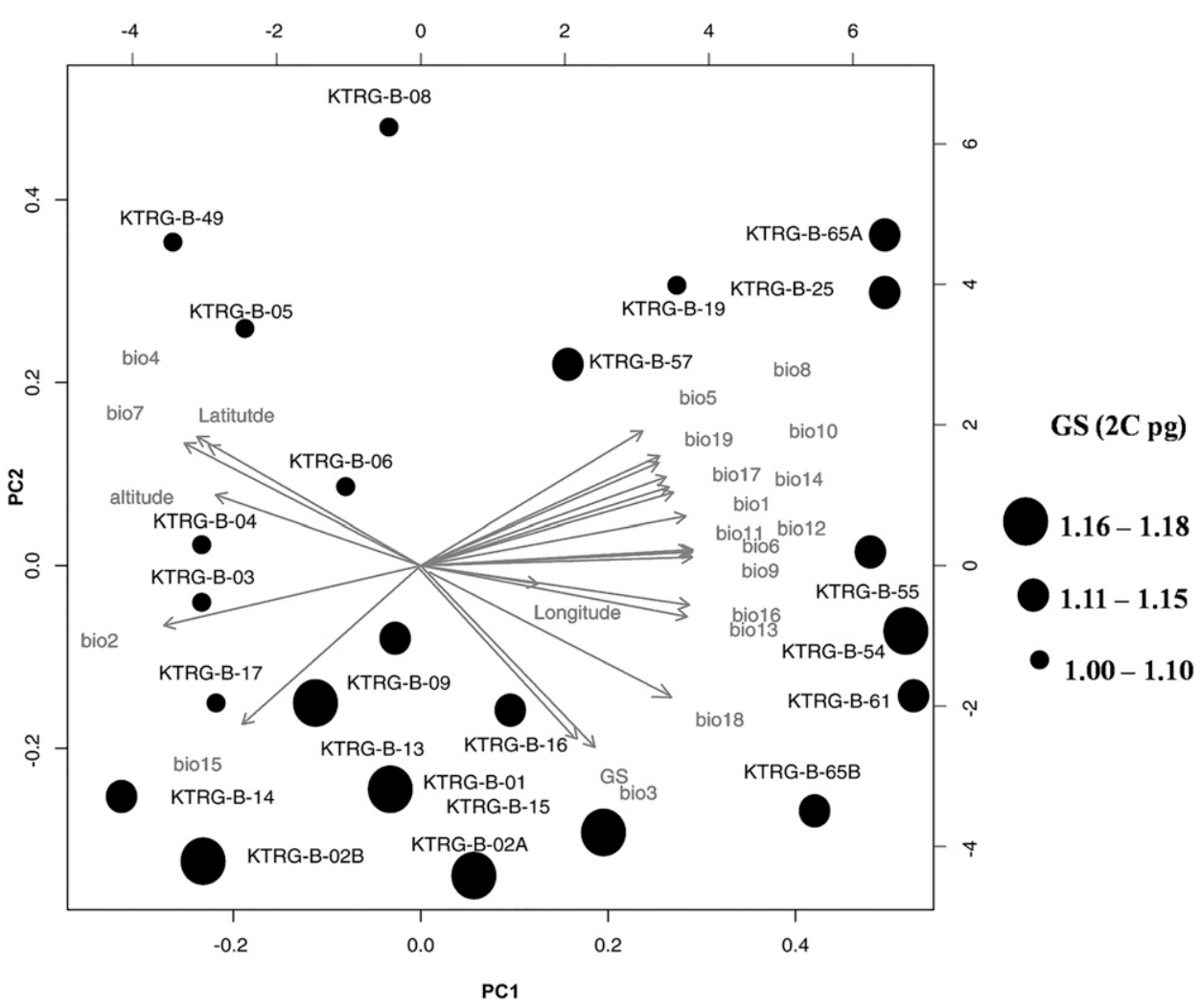

Fig. 3. Principal component analysis (PCA) of genome size (GS) and bioclimatic variables among 23 Brassica rapa var. rapa landraces included in this study. The arrows represented different models in the study. First principal component (PC1) and second principal component (PC2) explain 59.62\% and $22.77 \%$ of variance, respectively.

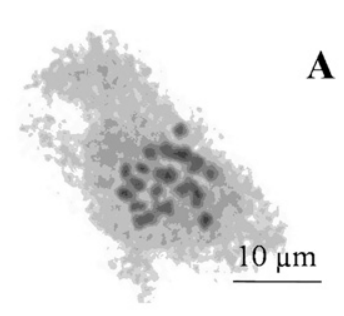

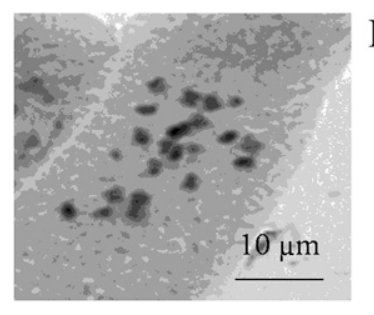

B

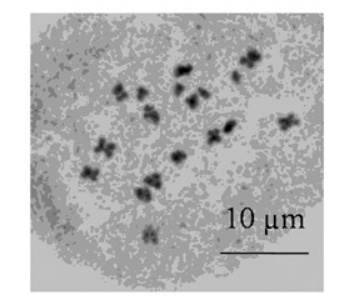

C

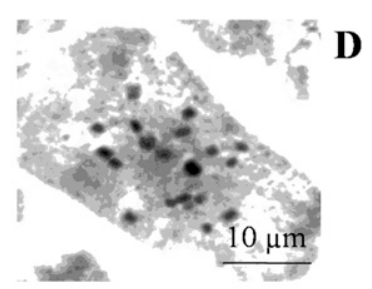

\section{$\mathbf{E}$}
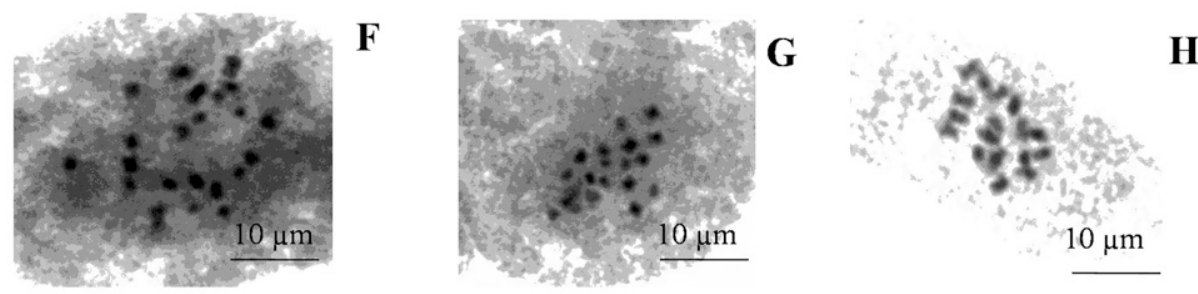

$\mathbf{H}$

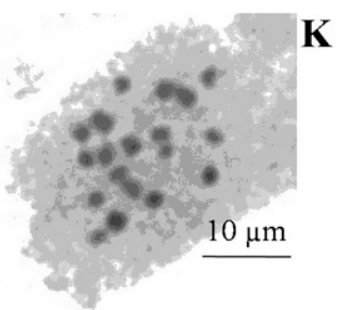

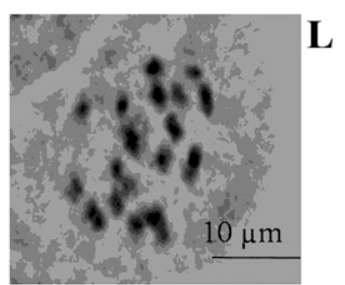

Fig. 4. Somatic chromosome at mitotic metaphase stage from root tips of Brassica rapa var. rapa landraces confirming euploidy with $2 n=2 x=20$ between QinghaiTibetan Plateau (QTP) and Yunnan Plateau (YP) landraces: (A) KTRG-B-03 [2C $=1.03 \pm 0.02 \mathrm{pg}(\mathrm{mean} \pm \mathrm{SE})],(\mathbf{B}) \mathrm{KTRG}-\mathrm{B}-05(2 \mathrm{C}=1.03 \pm 0.01 \mathrm{pg}),(\mathbf{C}) \mathrm{KTRG}-$ B-06 (2C $=1.03 \pm 0.01 \mathrm{pg}),(\mathbf{D})$ KTRG-B-08 $(2 \mathrm{C}=1.03 \pm 0.02 \mathrm{pg}),(\mathbf{E})$ KTRG-B-14 $(2 \mathrm{C}=1.11 \pm 0.01 \mathrm{pg}),(\mathbf{F})$ KTRG-B-17 $(2 \mathrm{C}=1.09 \pm 0.01 \mathrm{pg}),(\mathbf{G}) \mathrm{KTRG}-\mathrm{B}-01$ $(2 \mathrm{C}=1.18 \pm 0.02 \mathrm{pg}),(\mathbf{H})$ KTRG-B-02A $(2 \mathrm{C}=1.19 \pm 0.01 \mathrm{pg})$, (I) KTRG-B-09 $(2 \mathrm{C}=1.14 \pm 0.03 \mathrm{pg}),(\mathbf{J}) \mathrm{KTRG}-\mathrm{B}-13(2 \mathrm{C}=1.17 \pm 0.03 \mathrm{pg}),(\mathbf{K}) \mathrm{KTRG}-\mathrm{B}-15$ $(2 \mathrm{C}=1.17 \pm 0.03 \mathrm{pg})$, and $(\mathbf{L}) \mathrm{KTRG}-\mathrm{B}-16(2 \mathrm{C}=1.13 \pm 0.02 \mathrm{pg})$. The landraces were arranged by their location; that is, QTP $(\mathbf{A}-\mathbf{F})$ and $\mathrm{YP}(\mathbf{G}-\mathbf{L})$. 


\section{Literature Cited}

Achigan-Dako, E.G., J. Fuchs, A. Ahanchede, and F.R. Blattner. 2008. Flow cytometric analysis in Lagenaria siceraria (Cucurbitaceae) indicates correlation of genome size with usage types and growing elevation. Plant Syst. Evol. 276:9-19.

Bennett, M.D. and I.J. Leitch. 2011. Nuclear DNA amounts in angiosperms: Targets, trends and tomorrow. Ann. Bot. 107:467-590. Bennett, M.D., J.B. Smith, and R.I.L. Smith. 1982. DNA amounts of angiosperms from the Antarctic and south Georgia. Environ. Expt. Bot. 22:307-318.

Bennetzen, J.L., J. Ma, and K.M. Devos. 2005. Mechanisms of recent genome size variation in flowering plants. Ann. Bot. 95:127-132.

Benor, S., J. Fuchs, and F.R. Blattner. 2011. Genome size variation in Corchorus olitorius (Malvaceae s.1.) and its correlation with elevation and phenotypic traits. Genome 54:575-585.

Díez, C.M., B.S. Gaut, E. Meca, E. Scheinvar, S. Montes-Hernandez, L.E. Eguiarte, and M.I. Tenaillon. 2013. Genome size variation in wild and cultivated maize along altitudinal gradients. New Phytol. 199:264-276.

Doležel, J., M. Doleželova, and F.J. Novak. 1994. Flow cytometric estimation of nuclear DNA amount in diploid bananas (Musa acuminata and M. balbisiana). Biol. Plant. 36:351-357.

Doležel, J. and J. Bartoš. 2005. Plant DNA flow cytometry and estimation of nuclear genome size. Ann. Bot. 95:99-110.

Greilhuber, J., E.M. Temsch, and J.C.M. Loureiro. 2007. Nuclear DNA content measurement, p. 67-101. In: J. Doležel, J. Greilhuber, and J. Suda (eds.). Flow cytometry with plant cells. Wiley, Weinheim, Germany.

Guo, W., J. Yang, X.D. Sun, G.J. Chen, Y.P. Yang, and Y.W. Duan. 2016. Divergence in eco-physiological responses to drought mirrors the distinct distribution of Chamerion angustifolium cytotypes in the Himalaya-Hengduan mountains region. Front. Plant Sci. 7:1329.

Hijmans, R.J., S.E. Cameron, J.L. Parra, P.G. Jones, and A. Jarvis. 2005. Very high resolution interpolated climate surfaces for global land areas. Intl. J. Climatol. 25:1965-1978.

Kalendar, R., J. Tanskanen, S. Immonen, E. Nevo, and A.H. Schulman. 2000. Genome evolution of wild barley (Hordeum spontaneum) by BARE-1 retrotransposon dynamics in response to sharp microclimatic divergence. Proc. Natl. Acad. Sci. USA 97:6603-6607.

Kirik, A., S. Salomon, and H. Puchta. 2000. Species-specific doublestrand break repair and genome evolution in plants. EMBO J. 19:5562-5566.

Knight, C.A. and D.D. Ackerly. 2002. Variation in nuclear DNA content across environmental gradients: A quantile regression analysis. Ecol. Lett. 5:66-76.

Knight, C.A. and J.M. Beaulieu. 2008. Genome size scaling through phenotype space. Ann. Bot. 101:759-766.
Knight, C.A., N.A. Molinari, and D.A. Petrov. 2005. The large genome constraint hypothesis: Evolution, ecology and phenotype. Ann. Bot. 95:177-190.

Li, Y., S.N. Zhai, Y.X. Qiu, Y.P. Guo, X.J. Ge, and H.P. Comes. 2011. Glacial survival east and west of the Mekong-Salween divide in the Himalaya-Hengduan mountains region as revealed by AFLPs and cpDNA sequence variation in Sinopodophyllum hexandrum (Berberidaceae). Mol. Phylogenet. Evol. 59:412-424.

Liu, Y.F., L.X. Gong, L.L. Liu, H.F. Cai, X.Q. Wu, and Z. Ying. 2012. Determination on nutritional content of Tibetan turnip (Brassica rapa L.) and experimental study on improvement of mice hypoxia tolerance. Sci. Technol. Food Ind. 9:412-416.

Long, Q., F.A. Rabanal, D. Meng, C.D. Huber, A. Farlow, A. Platzer, Q. Zhag, B.J. Vilhjálmsson, A. Korte, V. Nizhynska, V. Voronin, P. Korte, L. Sedman, T. Mandáková, M.A. Lysak, Ü. Seren, I. Hellmann, and M. Nordborg. 2013. Massive genomic variation and strong selection in Arabidopsis thaliana lines from Sweden. Nat. Genet. 45:884-890.

Loureiro, J., E. Rodriguez, J. Dolezel, and C. Santos. 2007. Two new nuclear isolation buffers for plant DNA flow cytometry: A test with 37 species. Ann. Bot. 100:875-888.

McAllister, C., R. Blaine, P. Kron, B. Bennett, H. Garrett, J. Kidson, B. Matzenbacher, A. Glotzbach, and A.J. Miller. 2015. Environmental correlates of cytotype distribution in Andropogon gerardii (Poaceae). Amer. J. Bot. 102:92-102.

Messing, J., A.K. Bharti, W.M. Karlowski, H. Gundlach, H.R. Kim, Y. Yu, F. Wei, G. Fuks, C.A. Soderlund, K.F. Mayer, and R.A. Wing. 2004. Sequence composition and genome organization of maize. Proc. Natl. Acad. Sci. USA 101:14349-14354.

Piao, S., J. Fang, L. Zhou, Q. Guo, M. Henderson, W. Ji, Y. Li, and S. Tao. 2003. Interannual variations of monthly and seasonal normalized difference vegetation index (NDVI) in China from 1982 to 1999. J. Geophys. Res. Atmos. 108(D14):1-13.

R Core Team. 2013. R: A language and environment for statistical computing. R Foundation for Statistical Computing, Vienna, Austria.

Ruth, S., M. Renee, H. Li, Z. Fang, and Y. Wang. 2008. Spatial patterns of plant diversity and communities in Alpine ecosystems of the Hengduan Mountains, northwest Yunnan, China. J. Plant Ecol. 1:117-136.

Tatum, T.C., L. Nunez, M.M. Kushad, and A.L. Rayburn. 2006. Genome size variation in pumpkin (Cucurbita sp.). Ann. Appl. Biol. 149:145-151.

Wu, Y.Q., Z.J. Cui, G.N. Liu, D. Ge, J. Yin, Q. Xu, and Q. Pang. 2001. Quaternary geomorphological evolution of the Kunlun Pass areas and uplift of the Qinghai-Xizang (Tibet) Plateau. Geomorphology 36:203-216. 